\title{
Twins as Participants in Randomized Controlled Trials: A Review of Published Literature
}

\author{
Athula Sumathipala, ${ }^{1,2,3}$ Lisa Yelland, ${ }^{4,5}$ Debra Green, ${ }^{2}$ Tom Shepherd, ${ }^{1}$ Kaushalya Jayaweera, ${ }^{3}$ \\ Paulo Ferreira, ${ }^{6}$ and Jeffrey M. Craig ${ }^{7}$ \\ ${ }^{1}$ Research Institute for Primary Care \& Health Sciences, Keele University, Staffordshire, UK \\ ${ }^{2}$ South Staffordshire and Shropshire NHS Foundation Trust, Stafford, UK \\ ${ }^{3}$ Institute for Research and Development, Colombo, Sri Lanka \\ ${ }^{4}$ South Australian Health and Medical Research Institute, Adelaide, South Australia, Australia \\ ${ }^{5}$ School of Public Health, The University of Adelaide, Adelaide, South Australia, Australia \\ ${ }^{6}$ Faculty of Health Sciences, Musculoskeletal Health Research Group, University of Sydney, Sydney, New South Wales, \\ Australia \\ ${ }^{7}$ Murdoch Children's Research Institute and Department of Paediatrics, University of Melbourne, Royal Children's Hospital, \\ Melbourne, Victoria, Australia
}

\begin{abstract}
Monozygotic (MZ) and dizygotic (DZ) twins participate in research that partitions variance in health, disease, and behavior into genetic and environmental components. However, there are other innovative roles for twins in medical research. One such way is involving MZ and/or DZ twins in co-twin control-designed randomized controlled trials (RCTs). To our knowledge, no reviews have been conducted that summarizes the involvement of twins in RCTs. Therefore, we conducted a systematic literature search using the U.S. Clinical Trials Database, NHS electronic databases, MEDLINE, EMBASE, and PsychINFO for RCTs on publications involving MZ and/or DZ twins as RCT participants. Out of the 186,027 clinical trials registered in the U.S. clinical trial register ClinicaTrails.gov, only six RCTs used twins as participants. From 1,598 articles identified in our search, 50 peer-reviewed English language publications met our pre-defined inclusion criteria. Sample sizes for RCTs have ranged from a total number of participants from 2 to 1,162 ; however, 32 (64\%) studies had a sample size of 100 or less, and of those, $12(24 \%)$ had fewer than 10 . Both MZ and DZ twins have been recruited to the RCTs. In most instances (33/50) each twin from a pair were assigned to different study arms. Most of those studies included MZ twins only. Despite the methodological advantages, the use of $\mathrm{MZ}$ and $\mathrm{DZ}$ twins as participants in interventional RCTs appeared limited. The continuous development of innovative twin designs, especially RCTs, indicates that twin research can extend beyond the more widely recognized heritability estimates.
\end{abstract}

Keywords: twin research, RCT, randomization, sample size

Twins studies are best known for using the classical twin design, based on comparisons between the similarity of monozygotic (MZ) and dizygotic (DZ) twins to partition the variance in health, disease, and behavior into genetic and environmental components, predominantly using observational study designs (Boomsma et al., 2002; van Dongen et al., 2012)

Studies involving twins have become increasingly relevant due to the continuous work of twin registries and studies that have, collectively, amassed data and biological material on hundreds of thousands of twins, and have provided a valuable resource for studying complex genetic phenotypes and their underlying biology (Hur \& Craig, 2013). The availability of longitudinal data through the International Society of Twin Studies and International Network of Twin Registries (INTR) is also proving to be a valuable resource, not only for new studies but also for global collaborations (Buchwald et al., 2014). Data derived from a collaboration of 54 international twin cohort databases participating in the CODATwins Project is a prime example (Jelenkovic et al., 2015, 2016; Silventoinen et al., 2015, 2016; Yokoyama et al., 2016).

RECEIVED 6 October 2017; ACCEPTED 9 November 2017. First published online 28 December 2017.

ADDRESS FOR CORRESPONDENCE: Professor Athula Sumathipala, Research Institute for Primary Care \& Health Sciences Faculty of Medicine \& Health Sciences, Keele University, Staffordshire, ST5 5BG, UK. E-mail: a.sumathipala@keele.ac.uk 
Within-pair comparisons of phenotypically discordant $\mathrm{MZ}$ and DZ twin pairs through an observational co-twin controlled design can illuminate the non-shared environmental differences influencing human traits. However, such comparisons are arguably more efficient in intervention cotwin control studies using phenotypically concordant pairs, where one twin is randomly assigned to receive the intervention and the other twin acts as their control. A comparison between the co-twin control design in intervention and non-intervention studies, along with other novel utilities of this design, have been discussed in detail previously (Plomin \& Haworth, 2010).

The term 'intervention' refers broadly to any clinical maneuver offered to study participants that may have an effect on their health status (Jadad, 1998). Randomized controlled trials (RCTs) are considered the gold standard for testing interventions and 'the most rigorous way of determining whether a cause-effect relationship exists between treatment and outcome and for assessing the cost effectiveness of a treatment' (Sibbald \& Roland, 1998, p. 201). Random allocation provides participants the same chance of being assigned to each of the treatment groups (Altman, 1991). The purpose of random allocation is to ensure that the characteristics of participants are as similar as possible across treatment groups prior to the initiation of an intervention (baseline). If randomization is done properly, it reduces the risk of a serious imbalance in known and unknown factors that could influence the clinical course of the participants. Therefore, any significant differences between treatment groups in the outcome of interest can be attributed to the intervention and not to any unidentified factor(s).

However, involving MZ twins in a co-twin controldesigned RCT has advantages over the traditional RCT involving unrelated individuals. The co-twin control design can increase statistical power and provide perfect control for many of the potential confounding factors that could be imbalanced between treatment groups by chance, especially genetic makeup and age. The latter is particularly relevant to RCTs in children, where age matching is often a challenge. However, recruiting children in RCTs has ethical implications and needs especially strict ethical oversight.

DZ twins may also allow for matching due to shared environment as well as some genetic component and age, which can potentially justify the choice for recruiting DZ twins rather than siblings for a study. However, challenges such as teasing out individual factors in the context of complex interacting contributors may arise when using DZ twins in RCTs. It is also believed that the co-twin control RCT approach will have the additional advantage of requiring a relatively smaller sample size without reducing the statistical power (Plomin \& Haworth, 2010). However, if twins from the same pair are randomly assigned to the same treatment group or independently of each other, rather than to different treatment groups as in the co-twin control design, the benefits in sample size for an RCT involving twins may be lost (Yelland et al., 2017). Intervention studies involving twins do exist in the literature. One such trial testing the effect of Vitamin C intake on common cold symptoms found that the relative power of this design compared to an unpaired design could be 2-14 times stronger, which means an unpaired study design would require much larger sample sizes to detect the same effect (Carr et al., 1981; Martin et al., 1982).

However, in our opinion the full advantages and the rationale for involving twins in RCTs have not been adequately discussed or explored. Therefore, we carried out this review as the first step to identify studies using twins as participants for RCTs. In-depth analyses of the quality of individual studies and methodological issues of these studies or meta-analysis was not an aim of this review. In addition, a meta-analysis is not possible due to the heterogeneity of such studies and the varying interventions tested, resulting in no single treatment effect to be estimated. Instead, we aimed to identify all published material including RCTs involving only twins as participants up until 2015, using the selection criteria described below, and to summarize basic trial characteristics including sample size, inclusion criteria, whether trials include only MZ, DZ or both, and randomization method (i.e., whether same-pair twins were randomly assigned to the same treatment group independent of each other, or to different treatment groups in a co-twin control design).

\section{Materials and Methods}

A comprehensive search was carried out using the following databases: USA clinical trial (https://clinicaltrials. gov/), MEDLINE (1946-2015), EMBASE (1974-2015), and PsychINFO (1806-2015). We also performed an extensive search using PubMed (all publications until March 2015). Searches were confined to articles in English.

In the NHS library electronic database searches (MEDLINE, EMBASE, and PsychINFO), the search terms 'twins', 'randomi*', 'control'), and 'trial ${ }^{*}$ ' with Boolean operator AND was used in 'title and abstract'. Each database was searched individually using the search terms. This allowed for all alternative spelling (randomised and randomized), variations of control (control, controls, and controlled) and singular or plural trial (trial and trials) to be searched simultaneously. The search terms used for the PubMed search also took all of these variations into account (as per the NHS library search).

\section{Data Extraction}

Data were extracted by one reviewer (DG), and then checked independently by a second reviewer (AS). All publications identified in the searches were searched for duplicates. Eligibility for inclusion in the review was decided by DG and AS. Full versions of publications selected were obtained and reviewed independently by three authors (AS, 


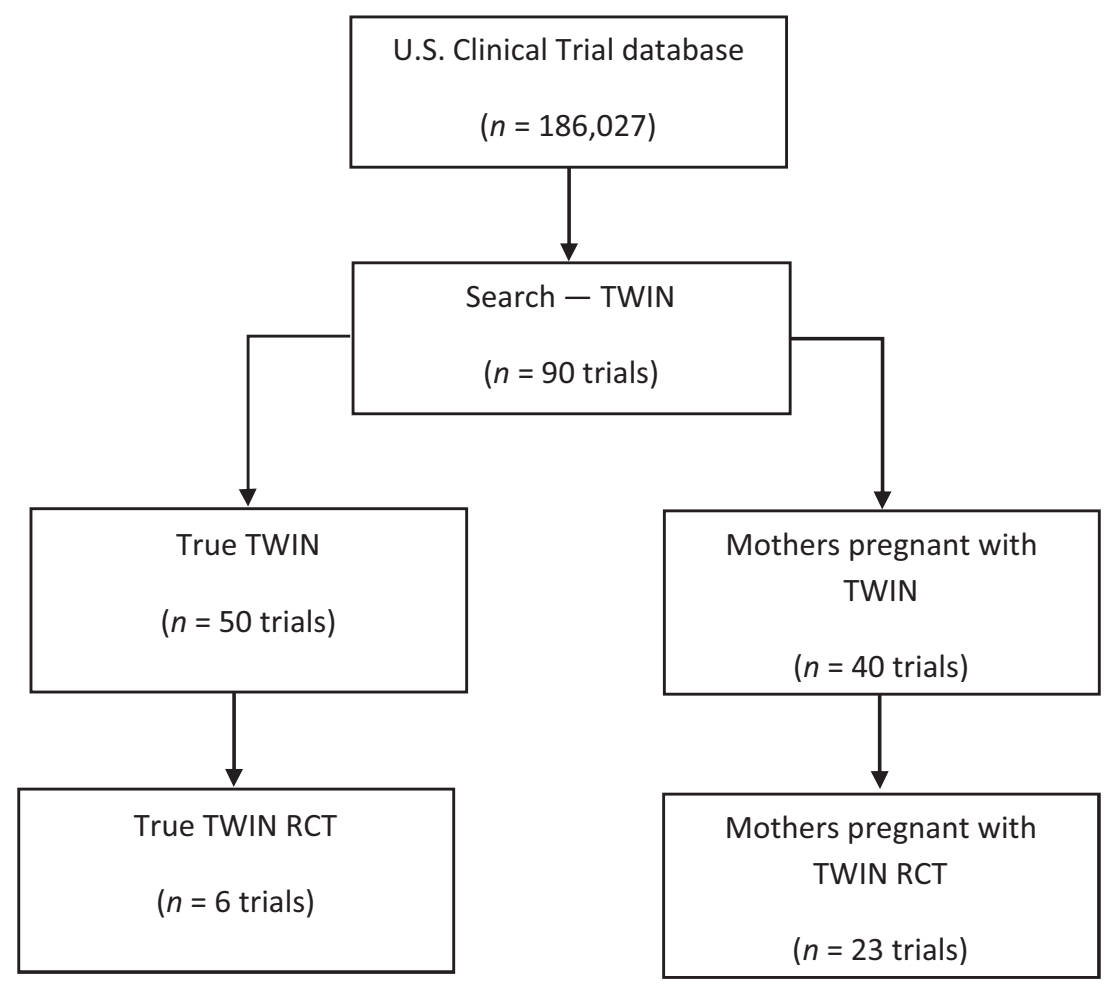

\section{FIGURE 1}

PRISMA diagram to illustrate the literature search process and the resulting number of reviewed articles from USA Clinical Trial database.

DG, and TS). Any discrepancy in judgments was resolved through consensus. Abstracts eligible for inclusion were confined to those reporting on a RCT that had used only twins as study participants but not mothers pregnant with twins. Information was extracted from each of the studies relating to authors, study location, database, twin registry (if any), condition, primary outcome, sample size, eligible age, eligible sex, zygosity, twin assignment (same treatment groups, different treatment groups, or independent allocation), masking, and control arm.

\section{Results}

\section{U.S. Clinical Trial Database}

The initial search using the U.S. clinical trial database (186,027 clinical trials registered) resulted in a total of 90 clinical trials. Of these, 50 studies were registered with twins as study participants, and 40 studies were registered with mothers who were pregnant with twins as study participants. Of the 90 twin studies, there were 29 RCTs. Of these, 23 had used mothers pregnant with twins as the study participants and only six studies had recruited twins as the study participants (Figure 1).

\section{Extended Search Using Other Databases}

After taking into account all duplicates and discounting papers including mothers pregnant with twins, the NHS li- brary searches gave a total of 51 papers on RCTs using twins as study participants; 47 from MEDLINE, and a further 4 papers not found in MEDLINE were found in EMBASE. No additional papers were found in PsychINFO.

PubMed searches gave an additional eight papers using twins as study participants that were not found in either the MEDLINE or EMBASE search. The search term 'Twins and Randomized Control Trial' found two papers; the term 'Twins and Randomised Controlled Trial' found five; and the term 'Twins and Randomised Control Trials' found one. The search terms 'Twins and Randomised Controlled Trial' and 'Twins and Randomised controlled trials' gave the exact same result when substituting the ' $s$ ' for a ' $z$ ' in the word 'randomised'. The full text of all 59 potentially relevant papers was examined in more detail, and 50 were consistent with the inclusion criteria for this review (Figure 2).

\section{Trial Characteristics}

Sample size and allocation of twins. The sample size varied greatly across the RCTs included in the review. Table 1 illustrates that $42 \%$ of RCTs had a sample size that fell within 10-100 twin pairs. Twenty four percent of the studies had a sample size smaller than 10 twin pairs as research participants. Therefore, the majority of studies had 100 twin pairs or fewer as participants. 


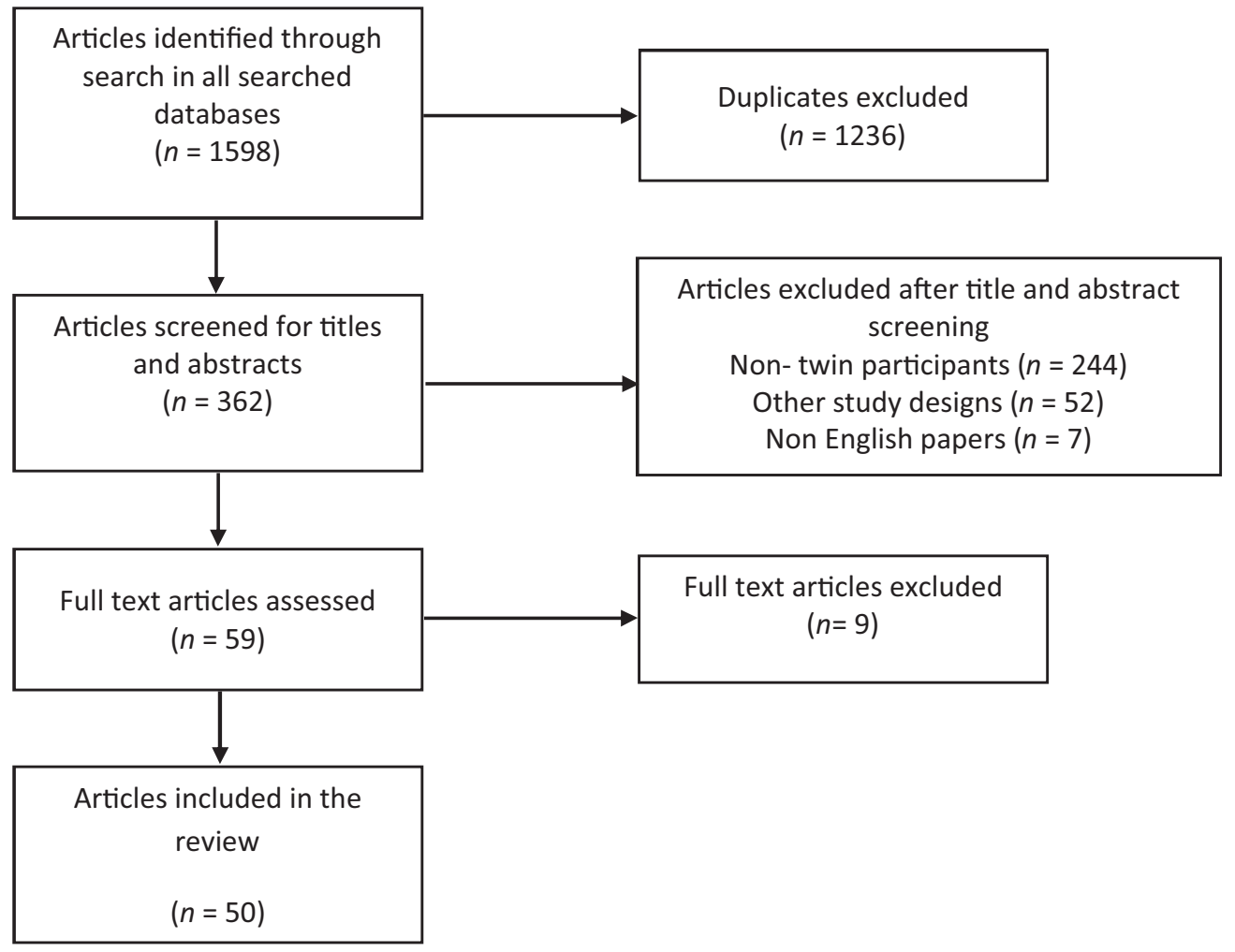

FIGURE 2

PRISMA diagram to illustrate the literature search process and the resulting number of reviewed articles found in the Medline, Psych INFO and EMBASE databases.

Zygosity and twin assignment across the randomized controlled trials. As illustrated in Table 1, 13 RCTs assigned both twins in a pair to the same study arm, 10 of which included both $\mathrm{MZ}$ and DZ twins, two included only $\mathrm{MZ}$ twins, and one included only DZ twins. This is in contrast to 33 RCTs where twin pairs were assigned to different study arms, of which 6 included both MZ and DZ twins, 24 included only $\mathrm{MZ}$ twins, and 3 included only $\mathrm{DZ}$ twins. In most instances $(33 / 50)$ the pair of twins had been assigned to different study arms, and most of these studies (24/33) had been with MZ twins.

Location of studies. The vast majority of the studies included in this review were conducted in the United States (21). The remaining studies were from Canada (5), Australia (3), United Kingdom (3), Finland (2), Germany (2), Greece (2), and one from each of the following countries; Bangladesh, Belgium, Dominican Republic, France, Hawaii, Hong Kong, India, Iran, Norway, Switzerland, Taiwan, and Thailand. A twin registry had been used to support recruitment in eight of the RCTs. These were the Australian Twin Registry (3), the Twin Research Registry, United Kingdom (3), the St. Thomas UK Adult Twin Registry (1), and the University of Washington Twin Registry (1). In the remaining 39 studies, there was no evidence of a twin registry being used to support recruitment. RCTs were diverse in nature, and areas included preterm birth nutrition, behavior, dental health, antiviral treatments, and cobedding of twins (twin infants sleeping together).

\section{Discussion}

RCTs involving only twins as participants are limited, as shown by this review. Only 50 studies met our inclusion criteria after a comprehensive search in clinical trials and NHS library databases, even across a wide range of disciplines. Out of the 186,027 clinical trials registered in the U.S. clinical trial register, only six RCTs used twins as participants. While it is impossible to determine how many RCTs had been reported through PubMed and other databases in total, it is clear that the number of twin RCTs is disproportionate to the number involving singletons. Therefore, it can be concluded that specifically using twins in RCTs is not common compared to using singletons.

\section{Randomization of Twins in a RCT}

When twins participate in a clinical trial, they may be randomized to the same treatment group, independent of each other, or to different treatment groups as in the co-twin control design. Our review found that most clinical trials 


\section{TABLE 1}

Characteristics of Randomized Controlled Trials With Twin Participants

\begin{tabular}{|c|c|}
\hline Characteristic & $\begin{array}{l}\text { Number (percentage) } \\
\text { of trials }(n=50)\end{array}$ \\
\hline \multicolumn{2}{|l|}{ Number of participants recruited } \\
\hline$<10$ & $12(24)$ \\
\hline 10-100 & $21(42)$ \\
\hline $101-250$ & $14(28)$ \\
\hline$>250$ & $3(6)$ \\
\hline \multicolumn{2}{|l|}{ Sex } \\
\hline Males only & $7(14)$ \\
\hline Females only & 9 (18) \\
\hline Males and females & $34(68)$ \\
\hline \multicolumn{2}{|l|}{ Age group } \\
\hline Infants only & $11(22)$ \\
\hline Child and adolescents only & $16(32)$ \\
\hline Adolescents and adults & $3(6)$ \\
\hline Adults only & $20(40)$ \\
\hline \multicolumn{2}{|l|}{ Twin assignment } \\
\hline Same treatment groups & $13(26)$ \\
\hline Different treatment groups & $33(66)$ \\
\hline Independent allocation & $3(6)$ \\
\hline Unclear & $1(2)$ \\
\hline \multicolumn{2}{|l|}{ Location } \\
\hline United States & $21(42)$ \\
\hline Canada & $5(10)$ \\
\hline Europe & $10(20)$ \\
\hline LMIC & $2(4)$ \\
\hline Other & $12(24)$ \\
\hline \multicolumn{2}{|l|}{ Twin recruitment method } \\
\hline Twin registry & $8(16)$ \\
\hline Other & $42(84)$ \\
\hline
\end{tabular}

used the co-twin control design with MZ twins. Therefore, it could be concluded that in the majority of studies, MZ twins had been used for perfect control of genetic variation between the treatment groups. Recent work suggests that twins and their parents have a strong preference for assigning both twins to the same treatment group, rather than using the co-twin control design (Bernardo et al., 2015). This has important implications for future RCTs conducted in twins, since recruitment may be more successful if both twins in a pair will receive the same treatment, although the impact of different methods of randomizing twins on the sample size must also be considered.

\section{The Impact of Twins on Sample Size and Power}

The sample size of trials included in our review ranged from 2 to 1,162 (Table 1 ) and in the majority of trials (64\%), the sample size was 100 or less. Although we did not attempt to assess whether the sample size was adequate for addressing the specific research question of each trial, this does raise the issue of whether small RCTs involving twins are adequately powered to detect meaningful treatment effects. One of the advantages of conducting RCTs in twins is that the sample sizes can be less compared to using non-twin RCTs (Carr et al., 1981; Martin et al., 1982; Miller et al., 1995). However, this will depend on how twins from the same pair are randomized. If the co-twin control design is used, such that one twin from each pair receives the intervention and the other acts as their control, the trial will have more power than a trial in singletons, and hence the sample size can be reduced. In contrast, if both twins are assigned to the same treatment group, the trial will have less power than a trial in singletons, thus requiring a larger sample size. This is due to the fact that comparisons of the intervention and control conditions must be made across twin pairs, rather than within twin pairs as in the co-twin control design. If twins from the same pair are randomized independently (ignoring that they are twins and treating as individuals), the trial will likely have similar power to a trial in singletons. Methods for calculating the sample size for trials involving twins only or a combination of singletons and twins have been discussed elsewhere (Yelland et al., 2017).

\section{International Collaborations Using Twin Registries}

We found that only $11 / 50$ (22\%) of the studies used twin registries for recruitment. One option particularly for multi-center RCTs with a relatively large sample size is international collaboration, as currently utilized in non-RCT twin research (Buchwald et al., 2014). The CODA Twins project was a classic example initiated in 2013 by identifying all twin projects in the world. It comprises 67 twin projects having data from both $\mathrm{MZ}$ and $\mathrm{DZ}$ twin pairs. The main sources used to identify the projects were a special issue of Twin Research and Human Genetics (Hur \& Craig 2013), and the participants of the INTR (Buchwald et al., 2014; van Dongen et al., 2012). The INTR is a platform for international collaborations and would be an excellent resource for large-scale multicenter clinical trials.

\section{Implications for Future Work and Directions}

To understand the potential benefits of the co-twin control design, it would be useful to compare the sample sizes of twin RCTs and non-twin RCTs required to detect the same effect size. The advantages and disadvantages of inclusion of both MZ and DZ twins in RCTs needs more in-depth discussion and are areas for future methodological research. Contamination between intervention and control twin participants allocated to different treatment groups (particularly in psychological interventions), especially among twins living together, will be an important issue to address.

\section{Limitation of the Review}

In-depth analyses of the quality of individual 50 studies qualified for the review and methodological issues of these studies were not done. We did not include a meta-analysis for this review.

\section{Conclusion}

Both MZ and DZ twins have been used in RCTs. However, in a majority of instances they have been MZ twins randomized to opposite arms of a RCT.

The continuous development and implementation of innovative twin designs in intervention studies, especially RCTs, indicates that twin research can extend beyond the 
more widely recognized heritability estimates toward the possibility of inference on causation.

\section{Acknowledgments}

JMC is supported by grants from the Australian $\mathrm{Na}$ tional Health and Medical Research Council (grant number 1079102), the Financial Markets Foundation for Children (grant number 2015-111), the Cerebral Palsy Alliance Research Foundation (grant number PG2316), and by the Victorian Government's Operational Infrastructure Support Program. LNY is supported by an Australian National Health and Medical Research Council Early Career Fellowship (ID 1052388).

\section{Conflict of Interest}

None.

\section{References}

Altman, D. G. (1991). Practical statistics for medical research. London, England: Chapman \& Hall.

Bernardo, J., Nowacki, A., Martin, R., Fanaroff, J. M., \& Hibbs, A. M. (2015). Multiples and parents of multiples prefer same arm randomization of siblings in neonatal trials. Journal of Perinatology, 35, 208-213.

Boomsma, D., Busjahn, A., \& Peltonen, L. (2002). Classical twin studies and beyond. Nature Reviews Genetics, 3, 872882.

Buchwald, D., Kaprio, J., Hopper, J. L., Sung, J., Goldberg, J., Fortier, I., ... Harris, J. R. (2014). International Network of Twin Registries (INTR): Building a platform for international collaboration. Twin Research and Human Genetics, 17, 574-577.

Carr, A., Martin, N., \& Whitfield, J. (1981). Usefulness of the co-twin control design in investigations as exemplified in a study of effects of ascorbic acid on laboratory test results. Clinical Chemistry, 27, 1469-1470.

Hur, Y.-M., \& Craig, J. M. (2013). Twin registries worldwide: An important resource for scientific research. Twin Research and Human Genetics, 16, 1-12.

Jadad, A. R. (1998). Randomised controlled trials: A user's guide. London, England: BMJ Books.

Jelenkovic, A., Hur, Y.M., Sund, R., Yokoyama, Y., Siribaddana, S. H., Hotopf, M., ... Silventoinen, K. (2016). Genetic and environmental influences on adult human height across birth cohorts from 1886 to 1994 . eLife, 5, 14.
Jelenkovic, A., Yokoyama, Y., Sund, R., Honda, C., Bogl, L. H., Aaltonen, S., ... Silventoinen, K. (2015). Zygosity differences in height and body mass index of twins from infancy to old age: A study of the CODATwins Project. Twin Research and Human Genetics, 18, 557570.

Martin, N., Carr, A., Oakeshott, J., \& Clark, P. (1982). Co-twin control studies: Vitamin C and the common cold. Progress in Clinical and Biological Research, 103, 365-373.

Miller, P. W., Mulvey, C., Martin, N., Miller, P., Mulvey, C., \& Martin, N. (1995). What do twins studies reveal about the economic returns to education? A comparison of Australian and U.S. findings. American Economic Review, 85, 586-599.

Plomin, R., \& Haworth, C. M. A. (2010). Genetics and Intervention Research. Perspectives on Psychological Science, 5, 557-563.

Sibbald, B., \& Roland, M. (1998). Understanding controlled trials. Why are randomised controlled trials important? BMJ, 316, 201.

Silventoinen, K., Jelenkovic, A., Sund, R., Honda, C., Aaltonen, S., Yokoyama, Y., \& Kaprio, J. (2015). The CODATwins Project: The cohort description of collaborative project of development of anthropometrical measures in twins to study macro-environmental variation in genetic and environmental effects on anthropometric traits. Twin Research and Human Genetics, 18, 348-360.

Silventoinen, K., Jelenkovic, A., Sund, R., Hur, Y.-M., Yokoyama, Y., Honda, C., ... Kaprio, J. (2016). Genetic and environmental effects on body mass index from infancy to the onset of adulthood: An individual-based pooled analysis of 45 twin cohorts participating in the COllaborative project of Development of Anthropometrical measures in Twins (CODATwins) study. The American Journal of Clinical Nutrition, 104, 371-379.

van Dongen, J., Slagboom, P. E., Draisma, H. H. M., Martin, N. G., \& Boomsma, D. I. (2012). The continuing value of twin studies in the omics era. Nature Reviews. Genetics, 13, 640-653.

Yelland, L. N., Sullivan, T. R., Price, D. J., \& Lee, K. J. (2017). Sample size calculations for randomised trials including both independent and paired data. Statistics in Medicine, 36, 1227-1239.

Yokoyama, Y., Jelenkovic, A., Sund, R., Sung, J., Hopper, J. L., Ooki, S., ... Silventoinen, K. (2016). Twin's birth-order differences in height and body mass index from birth to old age: A pooled study of 26 twin cohorts participating in the CODATwins Project. Twin Research and Human Genetics, 19, 112-124. 